\title{
The effect of group religious intervention on spiritual health and symptom reduction in patients with depression
}

\author{
Masoud Nikfarjam ${ }^{1}$, Kamal Solati ${ }^{1}$, Saeid Heidari-Soureshjani ${ }^{*}{ }^{*}$, Mohammadreza Nourmohammadi ${ }^{3}$, Seyed Yahya Kazemi ${ }^{3}$, \\ Amirgholi Jafari ${ }^{3}$, Maryam Omidi ${ }^{4}$ \\ 'Department of Psychiatry, Islamic Research Committee, Shahrekord University of Medical Sciences, Shahrekord, Iran \\ ${ }^{2}$ Deputy of Research and Technology, Sahrekord University of Medical Sciences, Shahrekord, Iran \\ ${ }^{3}$ Department of Islamic Teaching, Islamic Research Committee, Shahrekord University of Medical Sciences, Shahrekord, Iran \\ ${ }^{4}$ Islamic Research Committee, Shahrekord University of Medical Sciences, Shahrekord, Iran
}

*Corresponding Author: Saeid Heidari-Soureshjani, Tel: +989131833509, Email: heidari_1983@yahoo.com

\begin{abstract}
Background and aims: Depression is considered a disease which is associated with various complications including suicide and imposes high costs on the health systems. The present study aimed to determine the effectiveness of group religious intervention on spiritual wellbeing and symptom reduction in patients with depression.

Methods: In this clinical trial, 72 patients with depression were included using convenience sampling technique and then were randomly divided into patients with depression who underwent pharmacotherapy alone (group I) and those who underwent pharmacotherapy and religious intervention (group II). In addition, the religious group II participated in 5 90-minute sessions within three weeks and received the routine drug treatment. Then, a demographic questionnaire, Paloutzian and Ellison's Spiritual Well-Being scale, and Hamilton Depression scale were completed. Finally, the data were analyzed by the SPSS software, version 18 .

Results: There was no significant difference between the demographic characteristics of the patients $(P>0.05)$. However, the mean scores of religious and existential aspects of spiritual health, as well as the mean total score of spiritual health after the intervention were significantly higher in group II who received religious intervention compared to group I $(P<0.05)$. Conversely, the mean score of depression significantly decreased in groups I and II after the study $(P<0.001)$. In other words, after intervention, the mean score of depression was significantly lower in group II who received religious intervention compared to the group I $(P=0.038)$.

Conclusion: In general, religious intervention, reduced the symptoms of depression in patients in addition to increasing the level of the spiritual health of the patients.

Keywords: Religious intervention; Depression; Spiritual health
\end{abstract}

Received: 5 March 2018, Accepted: 25 April 2018, ePublished: 18 June 2018

\section{Introduction}

Spirituality is associated with certain concepts such as altruism, values, morality, and mental health, all of which are related to whatever is sacred and transcendental (1). In addition, spiritual health is determined by characteristics like sustainability in life, calmness, close relationship with God, and having a purpose in life, and gives the person's life meaning and concept $(2,3)$. Therefore, spiritual health is one of the integral parts of the health such that Eastern Mediterranean managers added it to the definition of health at their regional meeting (4). Mental health of individuals is another important aspect of health (5). Further, depression is regarded as one of the most common mental diseases related to different factors such as personal characteristics and even cultural issues which causes suicide and early death, as well as physical, psychological, and social illnesses for the affected person (6). According to the World Health Organization, around 350 million people at different ages suffer from depression, most of whom are women (7). There are various chemical treatments and psychotherapies to treat this type of disorder $(8,9)$ while religious treatment has not yet found a position in psychotherapy $(10,11)$. Accordingly, further studies are needed to clarify this issue. As a result, the current study sought to evaluate the effectiveness of group religious intervention on spiritual well-being and symptom reduction in patients with depression who referred to Imam Ali Clinic of Shahrekord during 2017.

\section{Materials and Methods}

The population of the present clinical trial study included 72 patients with depression who were enrolled by a convenience sampling technique. Then, patients with inclusion criteria were randomly included in the study. The inclusion criteria were the incidence of depression and attaining a score of at least 17 on the Hamilton Depression Scale (HAM-D) at the first administration (12). Furthermore, a diagnostic criterion for depression

(C) 2019 The Author(s); Published by Shahrekord University of Medical Sciences. This is an open-access article distributed under the terms of the Creative Commons Attribution License (http://creativecommons.org/licenses/by/4.0), which permits unrestricted use, distribution, and reproduction in any medium, provided the original work is properly cited. 
was used based on a structured clinical interview according to the criteria of the DSM-5 in order to ensure that the patients suffered from depression (13). Other inclusion criteria were lack of suffering from other mental illnesses, psychotic disorders, and severe types of these two diseases. Moreover, physical well-being and full consciousness for attending the sessions, and belief in God and the religious dimension of a man were considered for the purpose of inclusion. Conversely, Lack of providing consent to attend the sessions, the existence of suicidal thoughts, and disease progression were regarded as the exclusion criteria.

After obtaining informed and written informed consent from the participants, they were randomly assigned to two groups by using a random number table. Group I ( $\mathrm{n}=36)$ included patients with depression who received pharmacotherapy alone while group II $(n=36)$ encompasses patients with depression who underwent intervention and pharmacotherapy. Additionally, before the intervention, the level of depression and depression in patients who met the inclusion criteria were measured by an interview conducted by the therapist and then, the patients' level of depression was followed up for two months after the intervention.

In this study, a three-part questionnaire was used. The first part was a demographic questionnaire including items such as age, sex, marital status, economic status, level of education, place of residence, family history of the disease (grade 1), occupation, duration of depression, and depression. The second part was related to the Paloutzian and Ellison Spiritual Well-being Scale (1982) which includes 20 items regarding religious health $(\mathrm{n}=10)$ and the existential health of an individual $(n=10)$. The score on the sum of these two aspects represented the score of spiritual health ranging from 20 to 120 . In addition, the items of this questionnaire were rated on a 4-point Likerttype scale from Absolutely agree to Absolutely disagree. In 11 items (i.e., 3, 4, 7, 8, 10, 11, 14, 15, 17, 19, and 20), Absolutely disagree was assigned a value of 1 while in the remaining 9 items (i.e., 1, 2, 5, 6, 9, 12, 13, 16, and 18) this option was scored 6 . Finally, the spiritual health levels of the participants were divided into Low (20-40), moderate
(41-49), and high (100-120) categories. Further, Ellison and Smith obtained an internal consistency coefficient of 0.89 for the sum of the two aspects (14). Obviously, this questionnaire was nativized to Iranian people. In a study by Dastgheyb et al, the Cronbach $\alpha$ coefficient was estimated at 0.89 for this questionnaire (15).

The third part of the study instrument was the HAM-D. This scale contained 24 items and was used in various studies conducted in Iran $(16,17)$, the reliability of which was confirmed by Cronbach $\alpha$ coefficient of 0.74 (19). Different dimensions of depression such as behavioral, physical, cognitive, emotional, feeling guilty, hypochondria, gender issues, work, suicide, and sleep were examined. The score on each item ranged from 0 to 2 and 0 to 4 and the total score of the scale ranged from 0 to 50 . Based on this scale, respondents with a score of less than 17 were considered without depression. However, those with a score of 17-24, 25-30, as well as 31 and higher were considered to have mild, moderate, and severe depression, respectively (12).

Furthermore, the patient information was kept completely confidential and the names were stored as codes. The concurrence with clinical grading for psychiatric patients indicated moderate to high correlation coefficients and $r=0.72$ and CI of 0.55-0.96. Moreover, the correlation of Cronbach's Alpha test was obtained 0.73, 0.76 , and 0.76 by (HAM-D), Zong's Depression Scale, and MMPI depression scale, respectively (18). Group II attended religious sessions in five 90-minute sessions during 3 weeks and received a routine pharmacotherapy while group I only received a routine pharmacotherapy.

The intervention was implemented by a clergyman who worked in the medical university and held religious practices, lectures, group discussions, and slides. The content of the intervention sessions is provided in Table 1.

The data were collected after encoding and analyzed by the SPSS software, version 18 using descriptive statistics, independent $t$ test, paired $t$ test, and chi-square test.

Results

Totally, a number of 72 patients suffering from depression

Table 1. The Content of Educational Intervention in Patients With Depression

\begin{tabular}{|c|c|c|c|}
\hline Session & The Content of Group Religious Intervention & Equipment & Time (min) \\
\hline 1 & $\begin{array}{l}\text { The teaching of religious concepts and human relationship with God, God with the man, and the man with } \\
\text { the universe and nature, establishing prayer meetings and recommending to participate in congregational } \\
\text { prayers and religious rituals on a daily basis }\end{array}$ & Video projector & 90 \\
\hline 2 & $\begin{array}{l}\text { The role of hope, trust, forgiveness, patience, and resistance, and divine predestination in Islamic lifestyle; } \\
\text { holding prayer and repentance meetings and offering homework }\end{array}$ & Video projector & 90 \\
\hline 3 & $\begin{array}{l}\text { Reciting Quran and holy religious texts and thematic interpretation of these texts (with an emphasis on } \\
\text { Islamic lifestyle and family and social communication), and offering homework }\end{array}$ & Quran & 90 \\
\hline 4 & $\begin{array}{l}\text { Spiritual meditation and imagination, writing about and discussing spiritual feelings, resolving ambiguities, } \\
\text { worshiping God, and offering homework }\end{array}$ & Pen \& paper & 90 \\
\hline 5 & $\begin{array}{l}\text { Ethics and related concepts, healthy religious recreation, summarizing the contents and recommending the } \\
\text { continuation of the content implemented in the future }\end{array}$ & Video projector & 90 \\
\hline
\end{tabular}


were randomly enrolled in the present study and either received routine pharmacotherapy alone (group I, $\mathrm{n}=36$ ) or underwent religious intervention and pharmacotherapy (group II, $\mathrm{n}=36$ ). The mean age of the patients in the first and second groups was $36.99 \pm 10.20$ (18 to 56 years old) and $32 \pm 50.68$ (18 to 57 years old), respectively. Based on the results, no significant difference was observed in age, gender, marital status, economic status, education level, place of residence, family (first-degree relatives) history, occupation, and duration of depression between the two groups (Table 2).

Based on the results of the paired $t$ test, the mean scores of the religious aspect and existential aspect of spiritual well-being, as well as the total spiritual wellbeing after the intervention significantly increased in both groups $(P<0.001)$. Additionally, based on the results of the independent $t$ test, the mean scores of the religious aspect of spiritual well-being, existential aspect of spiritual well-being, and the total spiritual well-being after the intervention were significantly higher in group II who received religious intervention compared to group I $(P<0.05)$. However, the mean scores of depression significantly decreased in groups I and II $(P<0.001)$ and the mean score of depression after the intervention significantly reduced in group II who underwent religious intervention compared to group I $(P=0.038)$. The related

Table 2. The Frequency Distribution of Demographic Variables in Patients of Both Groups

\begin{tabular}{|c|c|c|c|c|c|c|}
\hline \multirow{2}{*}{ Variables } & & \multicolumn{2}{|c|}{ Group I } & \multicolumn{2}{|c|}{ Group II } & \multirow{2}{*}{ P Value } \\
\hline & & No. & $\%$ & No. & $\%$ & \\
\hline \multirow{2}{*}{ Sex } & Male & 18 & 50 & 13 & 36.1 & \multirow{2}{*}{0.234} \\
\hline & Female & 18 & 50 & 23 & 63.9 & \\
\hline \multirow{2}{*}{$\begin{array}{l}\text { Marriage } \\
\text { status }\end{array}$} & Married & 10 & 27.8 & 9 & 25 & \multirow{2}{*}{0.792} \\
\hline & Single & 26 & 72.2 & 27 & 75 & \\
\hline \multirow{5}{*}{$\begin{array}{l}\text { Educational } \\
\text { level }\end{array}$} & $\begin{array}{l}\text { Under high school } \\
\text { diploma }\end{array}$ & 4 & 11.1 & 5 & 13.9 & \multirow{5}{*}{0.297} \\
\hline & $\begin{array}{l}\text { High school } \\
\text { diploma }\end{array}$ & 9 & 25 & 12 & 33.3 & \\
\hline & Associate degree & 6 & 16.7 & 7 & 19.4 & \\
\hline & Bachelors degree & 16 & 44.4 & 8 & 22.2 & \\
\hline & MSc and higher & 1 & 2.8 & 4 & 11.1 & \\
\hline \multirow{6}{*}{ Job } & Employee & 3 & 8.3 & 5 & 13,9 & \multirow{6}{*}{0.554} \\
\hline & Laborer & 3 & 8.3 & 5 & 13.9 & \\
\hline & Self-employee & 15 & 41.7 & 10 & 27.8 & \\
\hline & Unemployed & 6 & 16.7 & 8 & 22.2 & \\
\hline & Housewife & 5 & 13.9 & 2 & 5.6 & \\
\hline & Student & 4 & 11.1 & 6 & 16.7 & \\
\hline \multirow{2}{*}{ Habitat } & Urban & 25 & 69.4 & 21 & 58.3 & \multirow{2}{*}{0.326} \\
\hline & Village & 11 & 30.6 & 15 & 41.7 & \\
\hline \multirow{4}{*}{$\begin{array}{l}\text { Economic } \\
\text { situation }\end{array}$} & Weak & 26 & 72.2 & 24 & 66.7 & \multirow{4}{*}{0.826} \\
\hline & Average & 6 & 16.7 & 8 & 22.2 & \\
\hline & Good & 2 & 5.6 & 3 & 8.3 & \\
\hline & Excellent & 2 & 5.6 & 1 & 2.8 & \\
\hline \multirow{2}{*}{$\begin{array}{l}\text { Anxiety } \\
\text { history }\end{array}$} & Yes & 8 & 22.2 & 8 & 22.2 & \multirow{2}{*}{1} \\
\hline & No & 28 & 77.8 & 28 & 77.8 & \\
\hline
\end{tabular}

data are presented in Table 3.

\section{Discussion}

The current study attempted to investigate the effectiveness of group religious intervention on spiritual well-being and symptom reduction in patients afflicted with depression. In this study, the mean scores of religious aspect and existential aspect of spiritual well-being, along with the total spiritual well-being after the intervention were significantly higher in group II compared to group I. Contrarily, it was found that the mean score of depression was significantly lower in group II after the intervention compared to the group I. Consistent with these results, Lyon et al found that a higher level of spiritual health indicates a higher quality of life while a lower level of depression and anxiety in adolescents with HIV (19). In addition, Dalmida et al reported a significant relationship between religious-spiritual practices and the reduction of depression symptoms in women with AIDS, which necessitates further research to clarify the ambiguous aspects of this issue (20). Further, a review was conducted to examine religious and spiritual interventions on several aspects of mental health care. Based on the findings obtained from clinical trials in the field of religious-spiritual interventions, it was revealed that the benefits of these interventions are manifested by reducing clinical symptoms, especially in anxiety and thus such interventions can be used as complementary therapies in health care filed (21). Furthermore, the results of a study implemented on Australian women demonstrated that there was a significant relationship between spiritual experiences and depression. Moreover, those who reported higher spirituality had higher social support whereas lower levels of depression (22). A review article indicated that although the comparison between and study of spirituality and religious studies on depression and anxiety was difficult due to the quality, various methods, and standard group IIs, paying more attention to religious beliefs and spiritual dimensions is inevitable among depressed and anxious outpatients, which should be considered a principle by the health care providers (23). Additionally, Kopacz examined the effect of spiritual health on suicide prevention in war injured and reported that depression-related factors such as suicidal thoughts were lower in people with higher levels of spiritual health (24). In addition, Mohammadi et al found that spiritual psychotherapy in patients with substance dependence disorder can reduce psychological problems such as depression and anxiety whereas it can improve the quality of life of these patients (25). Further, Bolhari et al studied the effectiveness of the spiritual group therapy on reducing depression, anxiety, and stress in women with breast cancer and observed that group spiritual therapy was significantly effective in alleviating the symptoms of depression while it improved spiritual health in these women. Furthermore, in a cohort study investigating 
Table 3. The comparison of mean scores of religious aspect, existential dimension of spiritual and total spiritual well-being, and depression scores between the two groups in pretest and post-test

\begin{tabular}{|c|c|c|c|c|}
\hline \multirow[t]{2}{*}{ Variable } & & \multirow{2}{*}{$\begin{array}{c}\text { Group I } \\
\text { Mean } \pm \text { SD }\end{array}$} & \multirow{2}{*}{$\begin{array}{c}\text { Group II } \\
\text { Mean } \pm \text { SD }\end{array}$} & \multirow{2}{*}{$P$ Value } \\
\hline & & & & \\
\hline \multirow{3}{*}{ Religious dimension of spiritual health } & Baseline & $33.8 \pm 5.6$ & $35.1 \pm 5.1$ & 0.335 \\
\hline & After intervention & $42.3 \pm 9.3$ & $47 \pm 6.1$ & 0.014 \\
\hline & $P$ value & $<0.001$ & $<0.001$ & - \\
\hline \multirow{3}{*}{ Existence dimension of spiritual health } & Baseline & $35.6 \pm 6.3$ & $35.5 \pm 6.7$ & 0.957 \\
\hline & After intervention & $43.2 \pm 8.1$ & $46.6 \pm 3.9$ & 0.027 \\
\hline & $P$ value & $<0.001$ & $<0.001$ & - \\
\hline \multirow{3}{*}{ Overall spiritual health } & Baseline & $69.4 \pm 10.3$ & $70.6 \pm 10$ & 0.621 \\
\hline & After intervention & $85.5 \pm 16.6$ & $93.6 \pm 8.4$ & 0.011 \\
\hline & $P$ value & $<0.001$ & $<0.001$ & - \\
\hline \multirow{3}{*}{ Depression } & Baseline & $27.3 \pm 5.6$ & $26.8 \pm 7.2$ & 0.732 \\
\hline & After intervention & $22.6 \pm 5.4$ & $19.7 \pm 5.9$ & 0.038 \\
\hline & $P$ value & $<0.001$ & $<0.001$ & - \\
\hline
\end{tabular}

the effect of spiritual intervention during pregnancy and determining its effect on postpartum depression, Mann et al indicated that attending religious ceremonies and strengthening the spiritual dimension of the studied women reduced the signs of postpartum depression, which was due to skill improvement for coping with the stress. As previously shown, the results of other studies demonstrated that spirituality of religion can have a preventative effect on many psychiatric disorders (28). However, the hypothesis which raised here was that religious and spiritual persons may be more depressed since they may feel guiltier or have a stronger feeling of committing a crime due to their illegitimate deeds (1). The argument should be considered in designing the curricula and educational protocols. However, religious interventions may be performed in various ways in accordance with the religious concepts of any religion. Clearly, religious programs should help promote self-esteem and self-efficacy of individuals and advise certain concepts such as patience and hope, which can result in resolving many psychiatric disorders, especially depression. Additionally, patients suffering from depression can be socially protected by participating in religious circles and being present in the community. In addition, they can contribute to promoting their spiritual and mental health by increasing their social interactions (29). What is certain is that the symptoms of the disease are nearly the same in the majority of humans depending on the type and severity of the disease while such symptoms should be further studied based on the type of religion. Finally, various religious practices have different effects on human mental states since religious beliefs and spiritual health may have a wide variety of definitions according to different religions.

\section{Conclusion}

In general, religious intervention, reduced the symptoms of depression in affected patients while increasing the level of the spiritual health of the patients. Therefore, such intervention can be employed as a complementary therapeutic approach to treat patients with depression. Furthermore, future studies are suggested to investigate the effect of interventions based on the severity of the disease and to study individuals in groups with similar disease severity. Additionally, interventions should be considered based on various divine religions whereas this should be performed by developing the relevant educational content and interventions. Eventually, the impact of these interventions can be addressed on other mental disorders.

\section{Conflict of interests}

None.

Ethical considerations

The current study was approved by Shahrekord University of Medical Sciences under the ethical code of IR.SKUMS.REC.1395.19.

\section{Acknowledgments}

This study was derived from a research project approved by the Research and Technology Deputy of Shahrekord University of Medical Sciences (approval no. 2132).

References

1. Koenig HG. Religion, spirituality, and health: the research and clinical implications. ISRN Psychiatry. 2012;2012:278730. doi: 10.5402/2012/278730.

2. Carven RF, Hirnle CJ, Hirnle C. Fundamental of nursing: human health and function. Philadelphia: Lippincott Williams \& Wilkins; 2003.

3. Dhar N, Chaturvedi S, Nandan D. Spiritual health scale 2011: defining and measuring 4 dimension of health. Indian J Community Med. 2011;36(4):275-82. doi: 10.4103/09700218.91329.

4. Nagase M. Does a Multi-Dimensional Concept of Health Include Spirituality? Analysis of Japan Health Science Council's Discussionson WHO's "Definition of Health"(1998). International Journal of Applied Sociology. 2012;2(6):71-7. doi: 10.5923/j.ijas.20120206.03.

5. Galderisi S, Heinz A, Kastrup M, Beezhold J, Sartorius N. Toward a new definition of mental health. World Psychiatry. 2015;14(2):231-3. doi: 10.1002/wps.20231. 
6. Kessler RC, Bromet EJ. The epidemiology of depression across cultures. Annu Rev Public Health. 2013;34:119-38. doi: 10.1146/annurev-publhealth-031912-114409.

7. World Health Organization (WHO). Depression. Genova: WHO; 2015.

8. Goldberg D. The "NICE Guideline" on the treatment of depression. Epidemiol Psichiatr Soc. 2006;15(1):11-5.

9. Sadock BJ, Sadock VA, Sussman N. Kaplan and Sadock's pocket handbook of psychiatric drug treatment. Lippincott Williams \& Wilkins; 2005.

10. Verghese A. Spirituality and mental health. Indian J Psychiatry. 2008;50(4):233-7. doi: 10.4103/0019-5545.44742.

11. Tuck I. A critical review of a spirituality intervention. West J Nurs Res.2012;34(6):712-35.doi:10.1177/0193945911433891.

12. Akhondzadeh S, Kashani L, Fotouhi A, Jarvandi S, Mobaseri $M$, Moin $M$, et al. Comparison of Lavandula angustifolia Mill. tincture and imipramine in the treatment of mild to moderate depression: a double-blind, randomized trial. Prog Neuropsychopharmacol Biol Psychiatry. 2003;27(1):123-7.

13. American Psychiatric Association. Diagnostic and statistical manual of mental disorders. Washington, DC: American Psychiatric Association; 2013.

14. Ellison C W, Smith J. Toward an integrative measure of health and well-being. J Psychol Theol. 1991;19(1):35-48. doi: 10.1177/009164719101900104.

15. Dastgheib Z, Gharlipour Z, Ghobadi Dashdebi K, Hoseini $F$, Vafaee R. Association of between mental health and spiritual health among students in Shiraz University. Adv Nurs Midwifery. 2014;24(84):53-9.

16. Nikfarjam M, Parvin N, Asarzadegan N. The effect of Lavandula angustifolia in the treatment of mild to moderate depression. Journal of Shahrekord University of Medical Sciences. 2010;11(4):66-73.

17. Ebrahimi A, Kheyr Z, Nasiri H, Barnamanesh A. Reliability, Validity, Factor and Discrimination Power Analysis of Young Mania Rating Scale among Unipolar, Bipolar Depression and Healthy People. Jundishapur Scientific Medical Journal. 2017;16(3):333-44. doi: 10.22118/jsmj.2017.49819.

18. Beck AT, Steer RA, Carbin MG. Psychometric properties of the Beck Depression Inventory: Twenty-five years of evaluation. Clin Psychol Rev. 1988;8(1):77-100. doi: 10.1016/0272-
7358(88)90050-5.

19. Lyon ME, Garvie P, He J, Malow R, McCarter R, D'Angelo LJ. Spiritual well-being among HIV-infected adolescents and their families. J Relig Health. 2014;53(3):637-53. doi: 10.1007/ s10943-012-9657-y.

20. Dalmida SG, Holstad MM, Diiorio C, Laderman G. Spiritual well-being, depressive symptoms, and immune status among women living with HIV/AIDS. Women Health. 2009;49(23):119-43. doi: 10.1080/03630240902915036.

21. Goncalves JP, Lucchetti G, Menezes PR, Vallada H. Religious and spiritual interventions in mental health care: a systematic review and meta-analysis of randomized controlled clinical trials. Psychol Med. 2015;45(14):2937-49. doi: 10.1017/ s0033291715001166.

22. Bennett KS, Shepherd JM. Depression in Australian women: the varied roles of spirituality and social support. J Health Psychol. 2013;18(3):429-38.doi:10.1177/1359105312443400.

23. Berry D. Does religious psychotherapy improve anxiety and depression in religious adults? A review of randomized controlled studies. Int J Psychiatr Nurs Res. 2002;8(1):875-90.

24. Kopacz MS. The spiritual health of veterans with a history of suicide ideation. Health Psychol Behav Med. 2014;2(1):34958. doi: 10.1080/21642850.2014.881260.

25. Mohammadi MR, Akbari AA, Hatami N, Mokri A, Kaviani $H$, Salmanian $M$, et al. Effectiveness of grouped spiritual psychotherapy on patients with opium using disorder. Hakim Res J. 2011;14(3):144-50.

26. Bolhari J, Naziri GH, Zamanian S. Effectiveness of spiritual group therapy in reducing depression, anxiety, and stress of women with breast cancer. Sociology of Women. 2012;3(1):85-115

27. Mann JR, McKeown RE, Bacon J, Vesselinov R, Bush F. Do antenatal religious and spiritual factors impact the risk of postpartum depressive symptoms? J Womens Health (Larchmt). 2008;17(5):745-55. doi: 10.1089/jwh.2007.0627.

28. Dabaghi P. Survey the role of spirituality and religion in health. Military Med J Islamic Rep Iran. 2009;4(1):1-4.

29. Nikfarjam M, Heidari-Soureshjani S, Khoshdel A, Asmand P, Ganji F. Comparison of spiritual well-being and social health among the students attending group and individual religious rites. World Fam Med J. 2017;15(8):160-5. 